\title{
Combination treatment for osteosarcoma with baculoviral vector mediated gene therapy (p53) and chemotherapy (adriamycin)
}

\author{
Sun U. Song ${ }^{1,3}$ and Frederick M. Boyce ${ }^{2}$ \\ ${ }^{1}$ Clinical Research Center, College of Medicine, Inha University, \\ 7-206, 3-Ga, Shinheung-Dong, Chung-Gu, Inchon 400-711, Korea \\ ${ }^{2}$ Department of Neurology, Massachusetts General Hospital, \\ Building 149, $13^{\text {th }}$ Street, Charlestown, MA 02129, U.S.A. \\ ${ }^{3}$ Corresponding author: Tel, +82-32-890-2460; \\ Fax, +82-32-890-2462; E-mail, sunuksong@inha.ac.kr
}

Accepted 23 March, 2000

Abbreviations: AcMNPV, Autographa californica multiple nuclear polyhedrosis virus; RSV, Rous sarcoma virus; CMVIE, cytomegalovirus immediate early; FACS, fluorescent activated cell sorter

\begin{abstract}
The insect baculovirus Autographa californica multiple nuclear polyhedrosis virus (AcMNPV) has been evaluated as a vector for gene delivery to human tumor cells. A human osteogenic sarcoma cell line, Saos-2, was found to be highly susceptible to infection with a baculoviral vector, with nearly $100 \%$ of Saos-2 cells being able to express a lacZ reporter gene after a brief exposure to the virus at a m.o.i. of $30 \mathrm{pfu} / \mathrm{cell}$. The production of $\beta$-galactosidase protein was 18-times greater than that in HepG2 cells which were previously thought to be the mammalian cells most susceptible to the baculovirus. The possibility of developing a baculovirus as a cytotoxic vector for p53-defective cancer was tested by destruction of Saos-2 cells $\left(\mathrm{p53}^{--}\right)$with a recombinant baculovirus containing the wild type p53 gene (BVp53) in vitro. The p53 baculovirus induced apoptotic cell death in tumor cells in a dose-dependent manner with $\sim 60 \%$ killing at an m.o.i. of 160 pfu/cell. Combined treatments of gene therapy (p53) and chemotherapy (adriamycin) resulted in synergistic and potent killing of the osteogenic sarcoma cells. For example, greater than $95 \%$ of Saos-2 cells were killed by the combination of BV-p53 (m.o.i. of 100) and adriamycin $(35 \mathrm{ng} / \mathrm{ml})$, whereas $\sim 50 \%$ and $\sim 55 \%$ cells were killed by BV-p53 and adriamycin alone, respectively. These results indicate that a baculoviral gene delivery vector can be used to efficiently target certain types of mammalian cells and the combination treatment of gene-therapy mediated by
\end{abstract}

a baculovirus and chemotherapy may enhance induction of apoptosis in cancer cells.

Keywords: combination therapy, baculoviral vector, p53 tumor suppressor gene, chemotherapeutic drug

\section{Introduction}

One new viral gene delivery vector developed in attempt to overcome the hurdles of current viral vector systems is based on the insect baculovirus Autographa californica multiple nuclear polyhedrosis virus (AcMNPV). The baculovirus has been thought to infect mainly insect cells until it has been shown that an expression of a reporter gene (lacZ) under the control of Rous sarcoma virus (RSV) promoter or cytomegalovirus immediate early (CMVIE) promoter was achieved in a human liver tumor cell line, HepG2 (Boyce and Bucher, 1996), and human hepatocytes (Hofmann et al., 1995), respectively. Although it was more recently shown that a recombinant baculovirus carrying a reporter gene under the control of strong CAG promoter which was composed of CMVIE enhancer and chicken $\beta$-actin promoter could infect nonhepatic cell lines, such as HeLa and COS7 (Shoji et al., 1997), hepatic cells have been thought to be the most susceptible cells to infection of baculovirus. Receptormediated endocytotic pathway was suggested for the cell uptake route of a baculovirus; however, the real mechanism of cell entry is still not well known. One of the advantages of using this insect virus for delivering a therapeutic gene to mammalian cells is that, unlike any other animal viruses, it does not usually replicate in mammalian cells, thus a baculoviral vector can be much safer than current viral vectors. Additional advantages of the baculoviral vector system include the ability to accommodate large inserts ( $>20 \mathrm{~kb}$ ), the ability to grow to high titers $\left(>10^{10} \mathrm{pfu} / \mathrm{ml}\right)$, and their ease of preparation. Although it is by now clear that AcMNPV can infect some mammalian cells and has useful features as a gene delivery vehicle, no one has shown the therapeutic use of a baculovirus yet.

The human p53 gene has been studied intensively because mutant forms of it have been found in about $50 \%$ of all tumors (Harris and Hollstein, 1993). p53 gene was first identified as a regulator for cell cycle in response to DNA damage. Later, it was found that expression of p53 induces not only stable growth arrest but also programmed cell death (apoptosis), depending 
on the level of p53 expression in a cell (Chen et al., 1996). If the level of p53 expression of a cell is less than the threshold level, the cell takes a growth arrest pathway, whereas the level is higher than the threshold level, it commits to an apoptosis pathway. Recently, Polyak et al. proposed a model for p53-induced apoptosis that suggests overexpression of p53 activates a group of genes involved in reduction-oxidation, leading to the formation of toxic reactive oxygen species and the oxidative destruction of mitochondrial components (Polyak et al., 1997). Promotion of cell cycle arrest and/or the induction of apoptosis by an exogenous expression of wild-type p53 have been observed in more than 100 cell lines and tissues with mainly adenoviral vectors due to its wide tissue tropism (Nielsen and Maneval, 1998). One potential problem with p53 adenovirus is a possibility of inactivation of p53 by a viral protein. For example, the $\mathrm{E} 1 \mathrm{~B}-55 \mathrm{kDa}$ protein of adenovirus type 5 is known to bind and inactivate p53, blocking apoptosis following p53 expression (Marcellus et al., 1996). A baculoviral vector system, relatively new but comparable or better to the adenoviral vector in some cell lines and tissues, has not been utilized in gene therapy with p53 tumor suppressor.

Surgery, chemotherapy, and radiation therapy are still main types of cancer treatments now. In the last several years, new approaches including gene therapy, cancer vaccines, immunotoxin therapy, antiangiogenic therapy have been developed and tested for their efficacy on a variety of different tumor models. More recently, the combination therapy of a conventional therapy and a new therapy has gained a basis as a more effective anticancer treatment than a single therapy alone (Fujiwara et al., 1994; Nguyen et al., 1996). Osteogenic sarcoma is one of the two most common bone tumors with Ewings sarcoma, occuring mainly during childhood and adolescence. Amputation and limb-sparing surgery have been main treatments before effectiveness of adjuvant chemotherapy has been proved recently. A regimen of combination of multiple drugs, such as combination of adriamycin, high-dose methotrexate and vincristine, is now considered as essential treatment for osteosarcoma (DeVita et al., 1997).

A human osteogenic sarcoma cell line, Saos-2, was found to show higher susceptibility than any other known permissive mammalian cells to infection of baculovirus. We demonstrate that Saos-2 tumor cells can be killed by p53-induced apoptosis, mediated with a recombinant baculovirus carrying p53 gene under the control of CMV promoter. The apoptotic death of Saos-2 cells was greatly enhanced by a combined treatment with an anticancer drug, adriamycin. These results demonstrate that an insect baculoviral vector can deliver a therapeutic gene to certain mammalian cells very efficiently and the combination treatment of p53-gene expression and a chemotherapeutic agent can enhance greatly the apoptosis of tumor cells.

\section{Materials and Methods}

\section{Preparation of virus}

The baculoviral transfer vector plasmid containing p53 open reading frame was constructed under the control of CMV promoter together with SV40 polyadenylylation signals in the background of pBacPAK9 (Clontech, Palo Alto, CA). The Bglll-Pacl fragment containing cytomegalovirus (CMV) promoter and internal ribosome entry sequence (IRES) regions derived from pCMV-Fab (Tac)protamine (Song and Marasco, 1999) is cloned into the pBacPAK9 plasmid cut with Bglll and Pacl restriction enzymes. The coding regions for $p 53$ and $\beta$-galactosidase were amplified by PCR using primers that contained Ascl and Notl sites and Nhel and Pacl sites respectively and cloned into the pBacPAK9-CMV-Fab (Tac)-protamine digested with the pairs of enzymes sequentially to generate $B V p 53-l a c Z$ transfer plasmid. LacZ gene inserted into the vector downstream of IRES region to utilize the expression of $\beta$-galactosidase protein in subsequent experiments was not active, confirmed by X-gal staining and western analysis (not shown), thus we could not utilize it in this experiment. Purification and amplification of recombinant baculoviruses were done as described (Boyce and Bucher, 1996).

\section{Western blot analysis and antibodies}

Mock- or virus-infected cell monolayers were lysed, separated by $10 \%$ SDS-PAGE, and transferred to nitrocellulose paper. They were probed with appropriate primary antibody before detection of reactive proteins using a horseradish peroxidase (HRP) secondary antibody and ECL detection method (Amersham, Piscataway, $\mathrm{NJ}$ ). Monoclonal anti-p53 antibody (Calbiochem, San Diego, CA) was used at a dilution of $1: 100$ for western blot analysis. Polyclonal anti-actin (Sigma, St. Louis, $\mathrm{MO}$ ) and anti- $\beta$-galactosidase antibodies (Chemicon, Temecula, CA) were used at a dilution of $1: 200$ and $1: 5,000$ respectively for western blot analysis.

\section{ELISA}

$3 \times 10^{5}$ cells/well were plated in a 6 -well plate a day before infection with BV-lacZ at an m.o.i. of 1, 10, and $100 \mathrm{pfu} /$ well. Cells were mock- or virus-infected with virus in $1 \mathrm{ml}$ of medium for $2 \mathrm{~h}$, and then the viruscontaining medium was removed and replaced with fresh medium. After $24 \mathrm{~h}$, the cells were washed three times with cold $1 \times \mathrm{PBS}$, scraped in $1 \mathrm{ml}$ of extract buffer (25 mM Tris pH 7.4, 0.1 mM EDTA), and transferred into an $1.5 \mathrm{ml}$ microcentrifuge tube. Cells were frozen in liquid nitrogen and thawed in a $37^{\circ} \mathrm{C}$ water bath three times. The extracts were spun down and the 
supernatants were removed to a fresh microcentrifuge tube. This supernatant was diluted with the extract buffer and used on ELISA assays and the remaining supernatant were kept at $-80^{\circ} \mathrm{C}$. For ELISA assay, 96-well plates were coated with rabbit polyclonal anti- $\beta$-galactosidase antibody $(20 \mu \mathrm{g} / \mathrm{ml}$, Chemicon, Temecula, CA) for $6 \mathrm{~h}$ to overnight at $4^{\circ} \mathrm{C}$ and rinsed twice with $1 \times$ PBS. The plates were blocked with 3\% BSA/PBS for 2 $\mathrm{h}$ at room temperature and rinsed twice with $1 \times \mathrm{PBS}$. A series of dilutions for $\beta$-galactosidase and supernatants from infected cells were added to the plates and incubated for $2 \mathrm{~h}$ at room temperature. The plates were rinsed four times with $1 \times$ PBS and mouse monoclonal anti- $\beta$ galactosidase (100 ng/ml, Roche, Postfach, Switzerland) antibody was added to each well and then incubated for $2 \mathrm{~h}$ at room temperature. The plates were rinsed four times with $1 \times$ PBS and anti-mouse lg-AP conjugated antibody (at $1: 1,000$, Roche, Postfach, Switzerland) that was preincubated with $0.1 \%$ normal rabbit serum were added and then incubated for $2 \mathrm{~h}$ at room temperature. The plates were then rinsed four times with $1 \times \mathrm{PBS}$ and three times with PNPP reaction buffer $(10 \mathrm{mM}$ Tris $\mathrm{pH}$ 9.5, $0.5 \mathrm{mM} \mathrm{MgCl}_{2}$ ). $100 \mu \mathrm{l}$ of PNPP substrate solution were added to each well and incubated for $3 \mathrm{~h}$ at $37^{\circ} \mathrm{C}$. $100 \mu \mathrm{l}$ of stop solution (0.1 mM EDTA) was added to each well and the $\mathrm{OD}_{405 \mathrm{~nm}}$ were measured using a microplate reader.

\section{FACS analysis}

$1-2 \times 10^{5}$ cells/well were seeded in a 6 -well plate a day before infection or combined treatment. Cells were mockor virus-infected with virus in $1 \mathrm{ml}$ of medium for $2 \mathrm{~h}$, and then the virus-containing medium was removed and replaced with fresh either straight or drug-containing medium. After $48 \mathrm{~h}$, the cells were trypsinized, spun down, and rinsed twice with wash buffer $(20 \mathrm{mM}$ Hepes $\mathrm{pH} 7.3,0.1 \% \mathrm{BSA}$ in PBS). The cells were then fixed in pre-cooled $70 \%$ ethanol at $4{ }^{\circ} \mathrm{C}$ overnight. The next day, the cells were washed again and then treated with RNase A $(5 \mu \mathrm{g} / \mathrm{ml})$ and propidium iodide (69 $\mathrm{mM}$ propidium iodide, $38 \mathrm{mM}$ sodium citrate) for $20 \mathrm{~min}$ at $37^{\circ} \mathrm{C}$. The samples were analyzed for DNA content using a Epics Elite ESP flow cytometer (Coulter, Miami, FL).

\section{MTT assay}

$1-2 \times 10^{5}$ cells were plated in a 6 -well plate a day before infection or combined treatment. Cells were mock- or virus-infected with virus in $1 \mathrm{ml}$ of medium for $2 \mathrm{~h}$, and then the virus-containing medium was removed and replaced with fresh either straight or drug-containing medium. After 5 days, $200 \mu \mathrm{l}$ of MTT solution $(5 \mathrm{mg} / \mathrm{ml}$ in PBS, sterilized by filtration) was added to the cells growing in $2 \mathrm{ml}$ of medium and incubated 4 more $\mathrm{h}$ at $37^{\circ} \mathrm{C}$. The reaction was stopped by adding $0.1 \mathrm{ml}$ of $10 \%$ SDS in $0.01 \mathrm{M} \mathrm{HCl}$ and then incubated further overnight at $37^{\circ} \mathrm{C}$. The absorbance at $595 \mathrm{~nm}$ was measured using a DU-650 spectrophotometer (Beckman, Fullerton, CA).

\section{Results}

To investigate if the insect baculovirus can be used as a gene delivery vector to p53-defective tumors, several p53-deficient cell lines were screened for their susceptibility to a recombinant baculovirus containing a lacZ reporter gene (BV-lacZ). A human osteogenic sarcoma cell line, Saos-2, was found to be highly susceptible to the infection of the recombinant baculovirus, with $100 \%$ cells staining blue with X-gal after exposure to $75 \mathrm{pfu} /$ cell of the virus (Figure 1D). Other p53-deficient cell lines, colon adenocarcinoma cell line (DLD-1) and glioblastoma cell line (T98G), were much less susceptible to the baculovirus (Figure 1F and H). The HepG2 cell line, which was previously thought to be the highly sus-

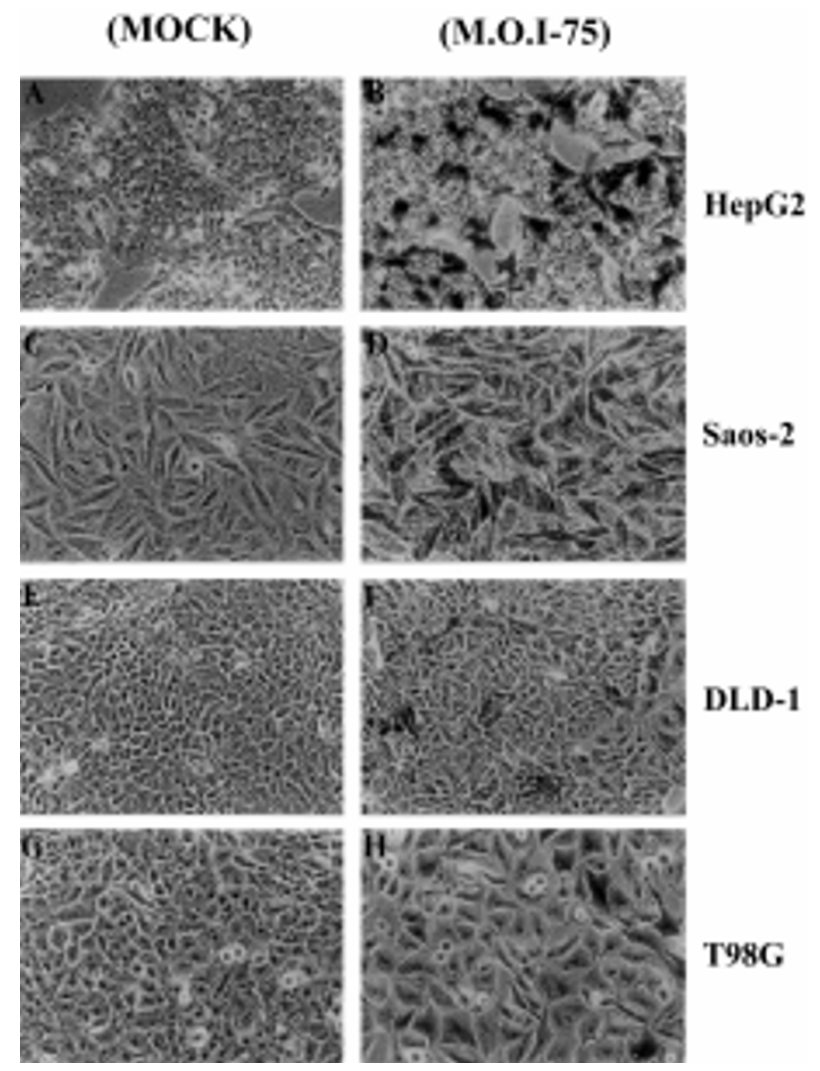

Figure 1. Screening for susceptibility of p53-defective cell lines to infection with a baculovirus. Three $p 53^{-1-}$ cell lines with a positive control HepG2 cell line were infected with a recombinant baculovirus containing lacZ gene under the control of the CMV promoter (BV-lacZ), and stained with X-gal 24 h post-infection. A and B, HepG2 cells; $C$ and D, Saos-2 cells; $E$ and $F$, DLD-1 cells; $G$ and $H, T 98 G$ cells. A, C, E, and G, mock-infected cells. B, $D, F$, and $H$, infected with BV-lacZ at an m.o.i. of 75 pfu/cell. The Saos-2 cell line was found to be highly susceptible to the baculovirus. 


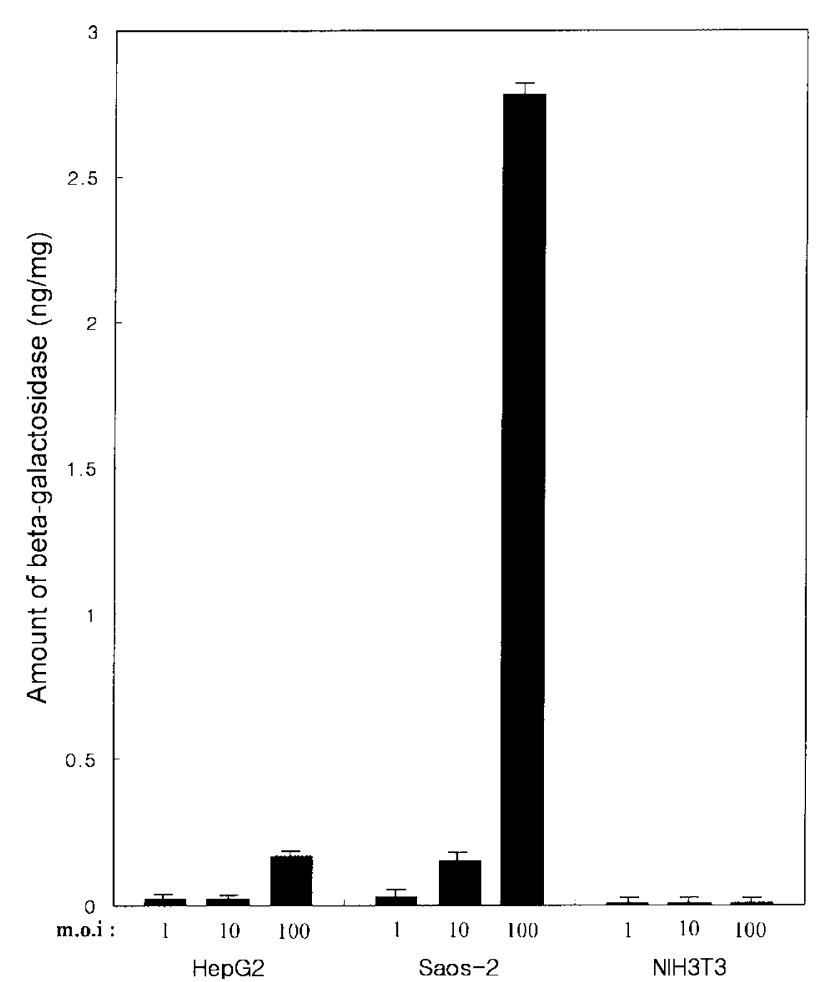

Figure 2. Quantitative comparison of $\beta$-galactosidase protein production in three cell lines. Amount of $\beta$-galactosidase protein expressed in HepG2, Saos-2, and NIH 3T3 cells was measured by ELISA assay $24 \mathrm{~h}$ after infection with a recombinant baculovirus containing lacZ gene (BV-lacZ) at an m.o.i. of 1,10 , or 100 pfu/cell. Values are expressed as amounts of $\beta$ galactosidase (ng) per total amount of cellular proteins $(\mathrm{mg})$. The value for mock-infected cells was subtracted in all cases. The amount of $\beta$ galactosidase protein produced in Saos-2 cells exceeds that of HepG2 cells which were thought to be the mammalian cells most susceptible to infection of a baculovirus. NIH3T3 cell line was used as a negative control. ceptible mammalian cell line, showed less X-gal staining (Figure 1B) with $60-70 \%$ expressing lacZ after exposure to the same amount of the virus. To confirm this result, a quantitative ELISA assay for $\beta$-galactosidase protein production was performed using extracts prepared from cells infected with varying doses of the lac $Z$ virus. The expression of $\beta$-galactosidase protein in Saos-2 cells infected with BV-lacZ was 18-times greater than that in HepG2 cells using equivalent doses at m.o.i. of either 10 or 100 pfu/cell (Figure 2). NIH 3T3 cells, which were previously shown to be relatively less susceptible to the baculovirus, showed a background level of $\beta$-galactosidase protein production (Figure 2).

To express p53 in Saos-2 cells, a recombinant baculovirus containing wild-type p53 gene under the control of the cytomegalovirus immediate-early promoter was constructed by cotransfection of the baculoviral transfer vector (Figure $3 \mathrm{~A}$ ) and a linear intact baculoviral genome into Sf21 insect cells. Production of p53 in Saos-2 cells was quantified by western analysis (Figure 3B). Mockinfected Saos-2 cell extracts showed no detectable p53 expression as previously reported (Lane 1, Figure 3B). Extracts from cells infected with BV-p53 at an m.o.i. of $100 \mathrm{pfu} / \mathrm{cell}$ showed high level of p53 production (Lane 2 , Figure 3B). The level of p53 expression peaked $\sim 24$ $\mathrm{h}$ after infection (not shown), consistent with earlier report of baculovirus mediated expression in mammalian cells. Extracts from HepG2 cells which were reported to contain wild-type p53 (Bressac et al., 1990) showed an apparently smaller p53 (Lane 3, Figure 3B), indicating that a mutant p53 protein may be present in this hepatocellullar carcinoma cell line.

To evaluate the possibility of developing a cytotoxic
A

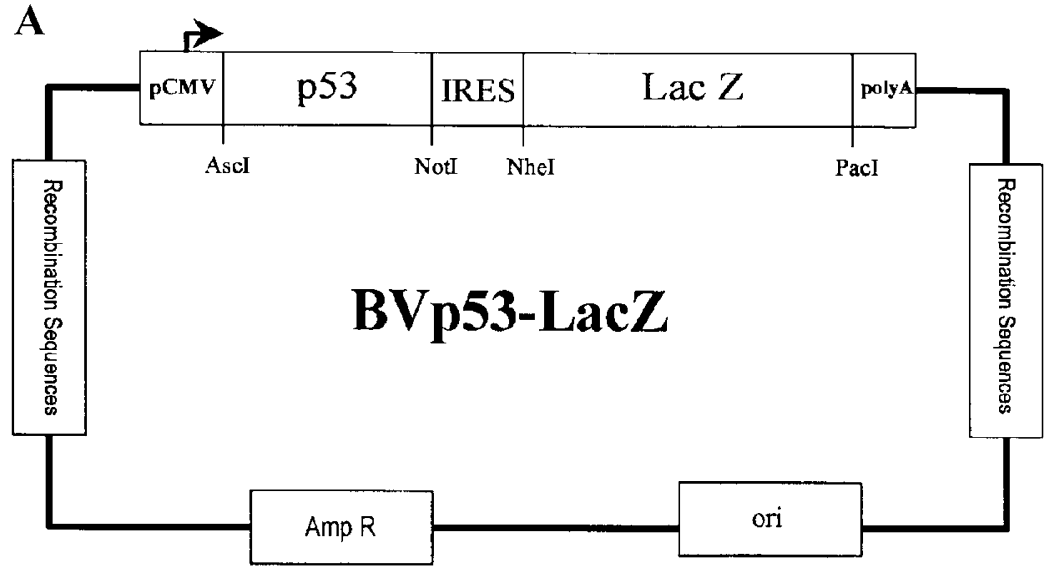

B

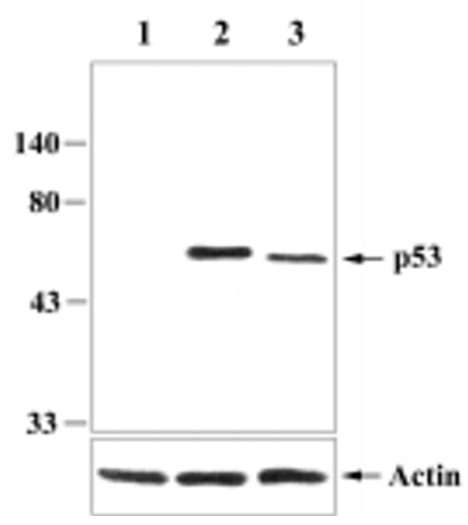

Figure 3. Expression of wild-type p53 protein in Saos-2 cells mediated by a recombinant baculovirus. (A) Schematic diagram of the baculoviral transfer plasmid vector encoding wild-type p53 and $\beta$-galactosidase protein under the control of cytomegalovirus IE promoter. The transfer plasmid vector DNA was inserted into the polyhedrin region of AcMNPV by homologous recombination. (B) Western blot of extracts from Saos-2 and HepG2 cells infected with the recombinant baculovirus (BV-p53) at an m.o.i. of 100 pfu/cell. Lane 1, mock-infected Saos-2 cells; lane 2, infected Saos-2 cells; lane 3, infected HepG2 cells. Cell extracts were prepared $24 \mathrm{~h}$ after infection, separated on 10\% SDS-PAGE gel, blotted into a nitrocellulose paper, and probed with antibodies against p53. Anti-actin antibody was used as an internal control for the amount of proteins loaded at each lane. Time course western of p53 expression showed that the production peaked around $24 \mathrm{~h}$ post infection (not shown). The expression of $\beta$-galactosidase protein was turned to be very weak, tested by western blot analysis and X-gal staining (not shown). 
A

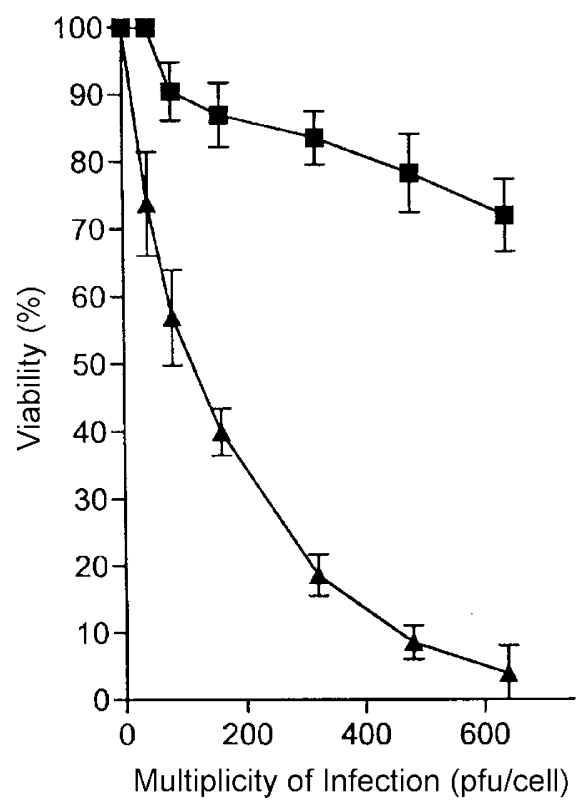

B

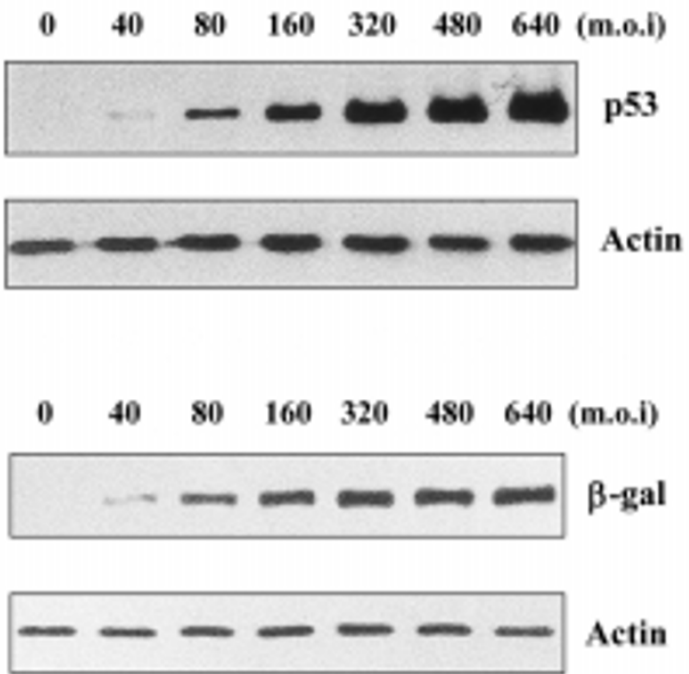

Figure 4. Viability of Saos-2 cells after infection with BV-p53 or BV-lacZ. (A) Viability of Saos-2 cells was measured by MTT assay 5 days after infection with BV-p53 or BV-lacZ at an m.o.i of 0, 40, 80, 160,320, 480, or 640 pfu/cell. Each point represents the average obtained from three independent experiments, with error bars representing the standard deviation. All values were normalized to the relative viability of the corresponding mock-infected controls from the same experiment. The $\mathrm{p53}$ baculovirus induced apoptotic cell death in a dose-dependent manner. Closed squares, cells infected with BV-lacZ viruses; closed triangles, cells infected with BV-p53 viruses. (B) Western blot of extracts from Saos-2 cells infected with BV-p53 or BV-lacZ at m.o.i. of 0, 40, 80, 160, 320, 480, or 640 pfu/cell. Cell extracts were prepared $24 \mathrm{~h}$ after infection, separated on 10\% SDS-PAGE, blotted into nitrocellulose paper, and probed with antibodies against p53, $\beta$-galactosidase, or control actin. The results indicate that there is no tight cellular inhibiting mechanism for the expression of these proteins.

vector based on baculovirus for p53-defective cancer cells, infection of Saos-2 cells with the BV-p53 viruses was performed followed by assays of cell death. The same number of Saos-2 cells $\left(1-2 \times 10^{5}\right)$ were infected with ascending doses of viruses, i.e., m.o.i. of $0,40,80$, $160,320,480$, or 640 pfu/cell. The viability of cells was measured by MTT assay 5days after infection with BVp53 or BV-lacZ as a control. The MTT assay is a sensitive and quantitative indicator of cell viability. The results showed that BV-p53 induced cell death in a dosedependent manner, for example, $26 \pm 2 \%, 60 \pm 1.5 \%$, and $91 \pm 5 \%$ with an m.o.i. of 40,160 , and 480 pfu/cell, respectively. Control BV-lacZ did not induce cell death in Saos-2 cells at the corresponding doses. However, the lacZ baculovirus did show some cytotoxic effect with high number of viruses (Figure 4A). Western blot analysis of cell extracts from infected Saos-2 cells revealed that expression of $\mathrm{p} 53$ and $\beta$-galactosidase protein were also increased in a dose-dependent manner, indicating that there is no tight cellular inhibiting mechanism for the expression of these proteins (Figure 4B).

To determine whether the p53-mediated cell death of Saos-2 cells occurs via apoptosis, Saos-2 cells were infected with BV-p53 or BV-lacZ and the DNA content of Saos-2 cells was analyzed by fluorescence activated cell sorting (FACS) $48 \mathrm{~h}$ after infection. BV-p53 gave a significant increase in the number of cells undergoing apoptotic cell death (cells with less than 2N DNA con- tent), compared with cells infected with the control BVlacZ (Figure 5). For example, Saos-2 cells infected with BV-p53 at an m.o.i of 100 or 400 pfu/cell showed $33.24 \%$ or $45.40 \%$ apoptotic cells, respectively (Figure $5 \mathrm{E}$ and $\mathrm{F}$ ), whereas $2.88 \%$ or $10.66 \%$ of cells were apoptotic with BV-lacZ, respectively (Figure $5 B$ and C). The analysis of Saos-2 cells infected with BV-p53 at an m.o.i. of $400 \mathrm{pfu} / \mathrm{cell}$ was hampered by detachment loss of the cells, and thus the determined value is likely to be underestimated.

It has been shown that the status of p53 genotype of tumor cells is an important determinant of tumor response

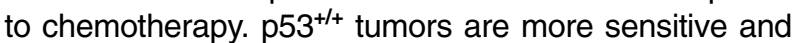
p $53^{-1-}$ tumors are less sensitive to chemotherapeutic agents, such as adriamycin, 5-fluorouracil, and etoposide (Lowe et al., 1993). To see whether p53-negative Saos-2 cells that have become p53-positive transiently by infection with BV-p53 turned to be sensitive to an anticancer drug, Saos-2 cells were treated with BV-p53 or BV-lacZ in combination with adriamycin. Viability testing after the treatment showed that BV-p53 (m.o.i. of 100) combined with adriamycin yielded more potent killing of Saos-2 cells than treatment with adriamycin alone (Figure 6A). BV-p53 in combination with $14 \mathrm{ng} / \mathrm{ml}$ or $35 \mathrm{ng} / \mathrm{ml}$ of adriamycin caused $\sim 80 \%$ and $~ 95 \%$ cell death, respectively, whereas adriamycin treatment alone yielded $\sim 10 \%$ and $\sim 55 \%$, respectively. In contrast, control BV-lacZ and adriamycin at equivalent doses generated 

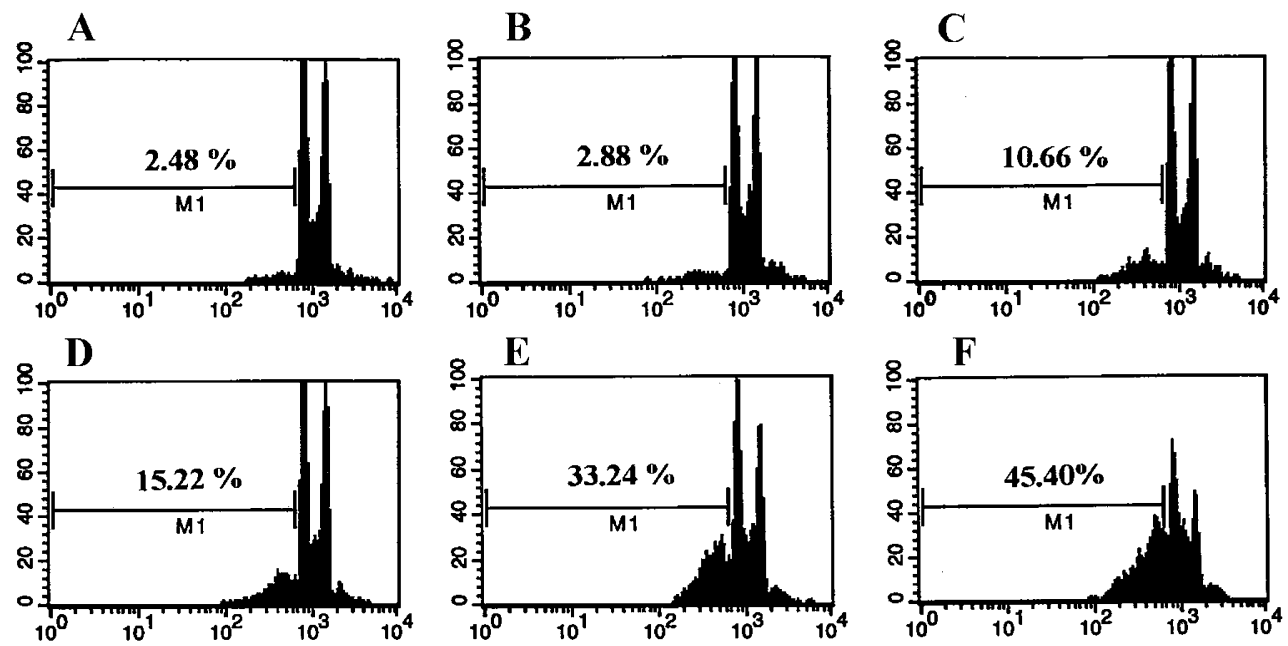

Figure 5. DNA content of Saos-2 cells infected with BV-p53 or BV-lacZ viruses. Cells were infected with the viruses at an m.o.i of 0, 50, 100, or 400 pfu/ cell. $48 \mathrm{~h}$ post infection, the cells were fixed and the DNA content was measured by propidium iodide staining and subsequent FACS analysis. (A), mockinfected; (B) and (C), infected with BV-lacZ at an m.o.i of 100 and 400 pfu/cell, respectively; (D)-(F), infected with BV-p53 at an m.o.i. of 50, 100, and 400 pfu/ cell, respectively. Cells undergoing apoptosis (less than $2 \mathrm{~N}$ DNA content) are indicated by the bracket (M1). Increased number of apoptotic cells was observed after treatment with BV-p53.

A

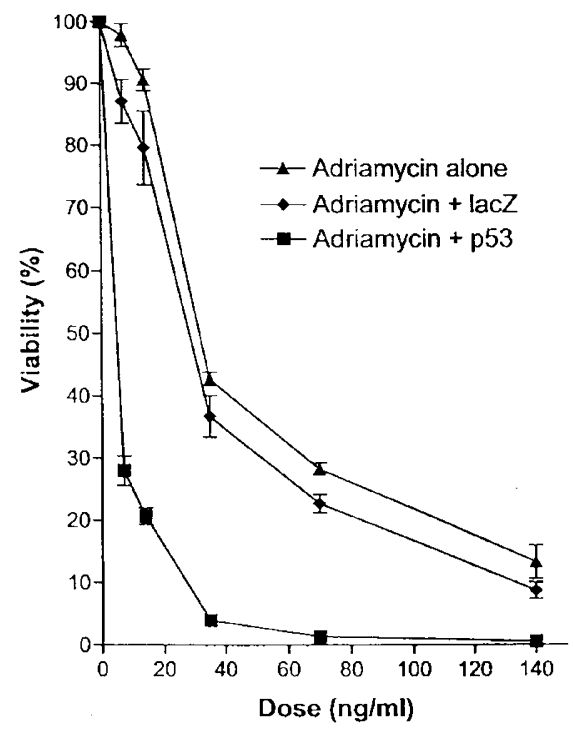

B
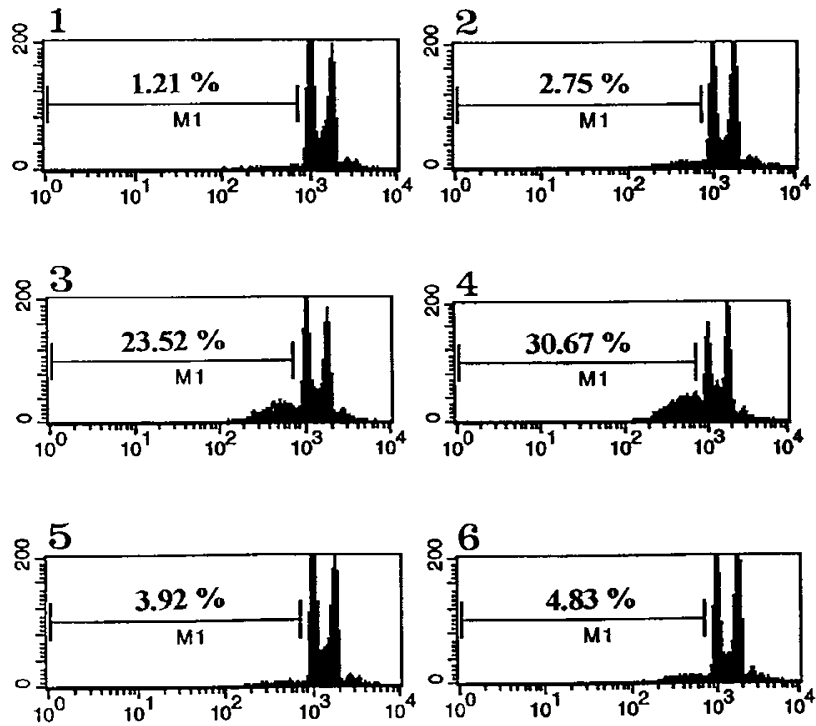

Figure 6. Viability of Saos-2 cells after combined treatment with recombinant baculovirus and adriamycin. (A) Viability of Saos-2 cells measured by MTT assay 5 days after combined baculovirus-adriamycin treatment or adriamycin treatment alone. A constant amount of virus (m.o.i. of 100) was used in all experiments with a series of different concentrations of adriamycin. The combined treatment with BV-p53 and adriamycin (closed squares) yie lded greater killing than treatment with adriamycin alone (closed triangles) or combined treatment with BV-lacZ and adriamycin (closed diamonds). (B) DNA content of Saos-2 cells treated with the combined treatment, virus alone, or adriamycin alone. Cells were treated with the viruses (m.o.i of 100) and/or adriamycin (20 ng/ml) and then fixed $48 \mathrm{~h}$ after the treatment and the DNA content was measured by FACS analysis. Cells undergoing apoptosis (less than $2 \mathrm{~N}$ DNA content) are indicated by the bracket (M1). Panel 1, mock-treated; panel 2, adriamycin alone; panel 3, BV-p53 alone; panel 4, BV-p53 and adriamycin; panel 5, BV-lacZ alone; panel 6. BV-lacZ and adriamycin.

$\sim 20 \%$ and $64 \%$ loss in viability, respectively. These values are not significantly higher than those with the single adriamycin treatment (Figure 6A).

To confirm cell death with the combined treatment occured via apoptosis, the DNA content of Saos-2 cells received a single or double treatment were analyzed by
FACS $48 \mathrm{~h}$ after the treatment. Adriamycin alone $(20 \mathrm{ng} /$ $\mathrm{ml}$ ) showed a value of apototic cell death which was just slightly higher than the background level (1 and 2, Figure $6 \mathrm{~B}$ ). The combined treatment with BV-p53 or BVlacZ and adriamycin gave $30.67 \%$ and $4.83 \%$ apoptotic cell death, respectively (4 and 6, Figure 6B). These 
results indicate strongly that the combination of p53baculovirus and adriamycin can give a synergistic effect on apoptosis of Saos-2 tumor cells.

\section{Discussion}

Here, a human osteogenic sarcoma cell line, Saos-2, is proven to be a highly susceptible one to infection with baculovirus. The induction of apoptotic tumor cell death with p53 baculovirus showed a dose-dependent manner in p53-defective Saos-2 cells. The cytotoxicity of the p53 baculovirus in the tumor cells was greatly increased with help of an anticancer drug, adriamycin. The 18-times higher susceptibility of Saos-2 cells than that of HepG2 cells which were thought to be the most susceptible to the infection with a baculovirus suggested that the tumor cell line would be an excellent in vitro model system for testing the possibility of developing p53 baculovirus as a cytotoxic vector for p53-deficient cancer cells. The dose-dependent manner with p53 baculovirus in killing the tumor cells indicates that the level of p53 expression has an important role in the p53-dependent apoptosis. Overproduction of p53 may overcome any viral or cellular anti-apoptotic activities. For example, baculoviral proteins, p35 and IAP, are known to inhibit the apoptosis of infected cells. In fact, the lack of inhibition of apoptosis in Saos-2 cells with the control lacZ baculovirus suggests that these anti-apoptotic baculoviral proteins are not active in Saos-2 cells.

It was apparent that the combined treatment of gene therapy (p53) and chemotherapy (adriamycin) gave much more severe cytotoxic effect to Saos-2 tumor cells than an individual therapy. In fact, the experiment of dosedependent single treatment with BV-p53 revealed a potential limitation of p53 gene therapy alone for cancer treatment. The single gene therapy required very high amount of viruses to get $>90 \%$ killing of Saos- 2 cells. The combined therapy showed a valuable advantage of lowering doses (several folds) of both virus and anticancer drug to achieve the same level of killing with the single therapy. This would be obviously a very important factor for safety when in vivo clinical trial is performed. Data with the combined treatment in Saos-2 cells support strongly the idea that combination therapy of gene therapy and chemotherapy would be beneficial for the in vivo treatment of cancers.

Comparison of previous studies (Boyce and Bucher 1996; Hofmann et al., 1995; Shoji et al., 1997; Wang et al., 1995; Hamada et al., 1996) and this study indicates that the efficiency of infection to HepG2 and Saos-2 cell lines with adenoviral or baculoviral vectors is highly comparable each other. Shoji et al. even showed a direct comparison using the same expression unit with the two systems. The results suggested that the level of expression of a reporter gene was equivalent in HepG2,
HeLa and COS7 cells (Shoji et al., 1997). Therefore these findings predict that adenoviral and baculoviral vector systems can be utilized equally well to some target tissues. It seems that so far the insect baculovirus shows a narrower tropism to mammalian cell lines and tissues than human adenovirus. This feature of baculovirus may be more beneficial in case that more targeted in vivo application is necessary. Since, like adenovirus, the insect baculovirus does not integrate into a host genome, a baculoviral vector system has potential advantages for cancer gene therapy to which a high level of transient expression of a therapeutic gene may be enough. Ease of producing high titer virus, high efficiency of infection, and high degree of tissue-specific targeting will make a baculoviral vector system be a very useful vehicle for in vivo cancer gene therapy including osteosarcoma and hepatoma.

It will be very important to find out the detailed mechanism of the synergistic effect of the combined treatment both in developing a novel approach of killing cancer cells and in elucidating a general mechanism of apoptosis of a cell. DNA damage caused by chemotherapy may not directly kill tumor cells, but rather induce an apoptosis program (Fisher, 1994). Multiple factors are likely to modulate "set point" of apoptosis induction. The set point may also be of great importance for the genesis and treatment of malignancy. The synergistic effect of the combined treatment may be resulted from an addition of either two p53-dependent pathways or separated p53-dependent and p53inpendent pathway. This study strongly suggests that combination therapy of different therapies would be more beneficial than single therapy for cancer treatment.

\section{Acknowledgment}

We thank Wayne Marasco and Abnor Mhashilkar for provision of pCMV-Fab(Tac)-protamine and p53 DNA. This work was supported by NRSA fellowship (CA7665602) from the National Cancer Institute to S. Song.

\section{References}

Boyce, F. M. and Bucher, N. L. R. (1996) Baculovirus-mediated gene transfer into mammalian cells. Proc. Natl. Acad. Sci. USA 93: 2348-2352

Bressac, B., Galvin, K. M., Liang, T. J., Isselbacher, K. J., Wands, J. R. and Ozturk, M. (1990) Abnormal structure and expression of p53 gene in human hepatocellular carcinoma. Proc. Natl. Acad. Sci. USA 87: 1973-1977

Chen, X., Ko, L. J., Jayaraman, L. and Prives, C. (1996) p53 levels, functional domains, and DNA damage determine the extent of the apoptotic response of tumor cells. Genes \& Dev. 


\section{0: $2438-2451$}

DeVita, V. T., Hellman S. and Rosenberg, S. A. (1997) Sarcomas of the soft tissues and bone. Cancer: Principles \& Practice of Oncology, Fifth Edition. Chap. 38: 1731-1852

Fisher, D. E. (1994) Apoptosis in cancer therapy: crossing the threshold. Cell 78: 539-542

Fujiwara, T., Grimm, E. A., Mukhopadhyay, T., Zhang, W. W., Owen-Schaub, L. B. and Roth, J. A. (1994) Induction of chemosensitivity in human lung cancer cells in vivo by adenovirus-mediated transfer of the wild-type p53 gene. Cancer Res. 54: 2287-2291

Hamada, K., Alemany, R., Zhang, W., Hitelman, W. N., Lotan, R., Roth, J. A. and Mitchell, M. F. (1996) Adenovirus-mediated transfer of a wild-type p53 gene and induction of apoptosis in cervical cancer. Cancer Res. 56: 3047-3054

Harris, C. C. and Hollstein, M. (1993) Clinical implication of the p53 tumor-suppressor gene. N. Eng. J. Med. 329: 13181327

Hofmann, C., Sandig, V., Jennings, G., Rudolph, M., Schlag, P. and Strauss, M. (1995) Efficient gene transfer into human hepatocytes by baculovirus vectors. Proc. Natl. Acad. Sci. USA 92: 10099-10103

Lowe, S. W., Ruley, H. E., Jacks, T. and Housman, D. E. (1993) p53-dependent apoptosis modulates the cytotoxicity of anticancer agents. Cell 74: 957-967

Marcellus, R. C., Teodoro, J. G., Charbonneau, R., Shore, G. C. and Branton, P. E. (1996) Expression of p53 in Saos-2 osteosarcoma cells induces apoptosis which can be inhibited by Bcl-2 or the adenovirus E1B-55 kDa protein. Cell Growth Differ. 7: 1643-1650

Nguyen, D. M., Spitz, F. R., Yen, N., Cristiano, R. J. and Roth, J. A. (1996) Gene therapy for lung cancer: enhancement of tumor suppression by a combination of sequential systemic cisplatin and adenovirus-mediated p53 gene transfer. J. Thorac. Cardiovasc. Surg. 112: 1372-1376

Nielsen, L. L. and Maneval, D. C. (1998) p53 tumor suppressor gene therapy for cancer. Cancer Gene Ther. 5: 52-63

Polyak, K., Xia, Y., Zweier, J. L., Kinzler, K. W. and Volgelstein, B. (1997) A model for p53-induced apoptosis. Nature 389: 300-305

Shoji, I., Aizaki, H., Tani, H., Ishii, K., Chiba, T., Saito, I., Miyamura, T. and Matsuura, Y. (1997) Efficient gene transfer into various mammalian cells, including non-hepatic cells, by baculovirus vectors. J. Gen. Virol. 78: 2657-2664

Song, S. U. and Marasco, W. A. (1999) Rapid establishment of myeloma cell lines expressing $\mathrm{Fab}$ (Tac)-protamine, a targetable protein vector, directed against the high affinity alpha chain of the human interleukin-2 receptor. Gene Targeting Vector Protocol, eds. Drs. Eric B. Kmiec and Dieter Gruenert, Humana Press, Inc., 157-166

Wang, J., Bucana, C. D., Roth, J. A. and Zhang, W. W. (1995) Apoptosis induced in human osteosarcoma cells is one of the mechanisms for the cytocidal effect of Ad5CMV-p53. Cancer Gene Ther. 2: 9-17 